\title{
Equilibrium Configurations of Point Vortices in Doubly Connected Domains
}

\author{
Alan R. Elcrat, Chenglie Hu and Kenneth G. Miller \\ Wichita State University \\ Wichita, KS 67260-0033, USA
}

\begin{abstract}
Point vortex flows of a steady, two dimensional, inviscid, and incompressible fluid are studied for doubly connected geometries. The Routh function is explicitly constructed, and equilibrium configurations of vortices are found by determining critical points numerically. The numerical computations make use of an analogue of the Schwarz-Christoffel transformation for doubly connected regions.
\end{abstract}

\section{Introduction}

Consider a two-dimensional motion of an incompressible and inviscid fluid in a multiply connected two-dimensional region $R$. The boundary of $R$ is assumed to consist of piecewise analytic curves $\Gamma_{k}(k=0,1, \ldots, m)$ with the external boundary, if there is one, denoted by $\Gamma_{0}$. The motion is assumed to be irrotational except for a number of free point vortices with strength $\kappa_{i}$ at points $P_{i}(i=1,2, \ldots, n)$. We assume further that all boundaries are fixed, the motion is steady at infinity, and no external forces act. Therefore the streamfunction for the motion does not depend on the time explicitly and the constant circulation around boundaries is subject to Kelvin's theorem.

If $u_{i}$ and $v_{i}$ are the components of the velocity of the $i$ th vortex, the Kirchhoff-Routh path function $W=W\left(z_{1}, z_{2}, \ldots, z_{n}\right)$ is a function depending on the coordinates $z_{i}=\left(x_{i}, y_{i}\right)$ of vortex positions such that

$$
\kappa_{i} \frac{d x_{i}}{d t}=\kappa_{i} u_{i}=-\frac{\partial W}{\partial y_{i}}, \quad \kappa_{i} \frac{d y_{i}}{d t}=\kappa_{i} v_{i}=\frac{\partial W}{\partial x_{i}}
$$

where $\kappa_{i}$ are strengths of the vortices. The vortex positions $z_{1}^{0}, z_{2}^{0}, \ldots, z_{n}^{0}$ are in equilibrium if

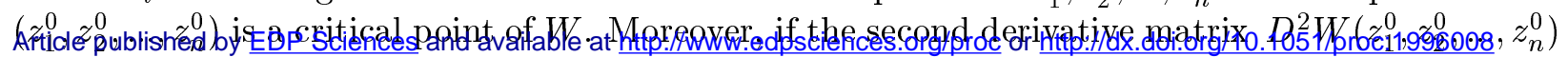
is positive definite, then this equilibrium configuration is stable as a solution of the Hamiltonian system (1).

We will use an expression for $W$ following Lin [4]. As in [4] we use a Green's function $G(z, Z)$ defined by the following conditions:

$$
\begin{aligned}
\Delta G:= & \frac{\partial^{2} G}{\partial x^{2}}+\frac{\partial^{2} G}{\partial y^{2}}=0 \quad \text { for } z=(x, y) \neq Z=(X, Y), \\
G \sim & \log \left(\sqrt{(x-X)^{2}+(y-Y)^{2}}\right) \text { as }(x, y) \rightarrow(X, Y), \\
G= & A_{k}(Z), \quad z \in \Gamma_{k}(k=1,2, \ldots, m), \\
& \left(A_{k}(Z) \text { unprescribed constants with respect to } z\right)
\end{aligned}
$$




$$
\begin{gathered}
\int_{\Gamma_{k}} \frac{\partial G}{\partial n} d s=0,(k=1,2, \ldots, m) \\
G(z, Z)=0, z \in \Gamma_{0} .
\end{gathered}
$$

If $R$ is unbounded, we also require $G(z, Z)=O(1)$ over all sufficiently large circles centered at $Z=(X, Y)$. We assume here that $\Gamma_{k}$ is piecewise analytic.

If the circulations around $\Gamma_{k}$ and velocities at infinity are given, the streamfunction for the flow with point vortices located at $z_{i}(i=1,2, \ldots, n)$ is

$$
\Psi\left(z ; z_{1}, \ldots, z_{n}\right)=\Psi_{0}(z)+\sum_{i=1}^{n} \kappa_{i} G\left(z, z_{i}\right) .
$$

Here $\Psi_{0}$ is the streamfunction of the potential flow in $R$ in the absence of vortices (for the given circulations around $\Gamma_{k}$ and velocities at infinity). It is shown in [4] that the Kirchhoff-Routh path function is given by

$$
W\left(z_{1}, \ldots, z_{n}\right)=\sum_{i=1}^{n} \kappa_{i} \Psi_{0}\left(z_{i}\right)+\frac{1}{2} \sum_{i \neq j} \sum \kappa_{i} \kappa_{j} G\left(z_{j}, z_{i}\right)+\frac{1}{2} \sum_{i=1}^{n} \kappa_{i}^{2} g\left(z_{i}, z_{i}\right),
$$

where

$$
g(z, Z):=G(z, Z)-\log (|z-Z|) .
$$

Under a conformal mapping $z=f(w), f: R_{w} \rightarrow R_{z}$, which transplants the motion in the $w$-plane to a motion in the $z$-plane, the Routh function for the new motion is given by

$$
\tilde{W}\left(z_{1}, \ldots, z_{n}\right)=W\left(w_{1}, \ldots, w_{n}\right)-\sum_{i=1}^{n} \frac{\kappa_{i}^{2}}{2} \log \left(\left|\frac{d f}{d w}\right|_{w=w_{i}}\right),
$$

where $z_{i}=f\left(w_{i}\right)$. The streamfunction $\tilde{\Psi}$ for the new motion in the $z$-plane satisfies

$$
\tilde{\Psi}\left(z ; z_{1}, \ldots, z_{n}\right)=\Psi\left(w ; w_{1}, \ldots, w_{n}\right)
$$

provided that the inner and outer boundaries of $R_{w}$ are mapped to inner and outer boundaries of $R_{z}$ respectively.

Any doubly connected polygonal region in the $z$-plane can be mapped to an appropriate annulus in the $w$-plane with the following version of the Schwarz-Christoffel map,

$$
f(w)=f\left(w^{*}\right)+C \int_{w^{*}}^{w} \prod_{k=1}^{M}\left[\theta\left(\frac{w}{\mu w_{0 k}}\right)\right]^{\alpha_{0 k}-1} \prod_{k=1}^{N}\left[\theta\left(\frac{w}{\mu w_{1 k}}\right)\right]^{\alpha_{1 k}-1}
$$

where $C$ is a complex constant. The definition of the $\theta$-function is given by

$$
\theta(w)=\prod_{d=1,3, \ldots}^{\infty}\left(1-\mu^{d} w\right)\left(1-\mu^{d} w^{-1}\right) .
$$

The remaining notation in (11) is illustrated in Fig. 1. Based on an earlier work of Daeppen [1], we have developed a Fortran package called DSCPACK to implement (11). Details concerning DSCPACK are in [3].

We give an analytical expression for the Green's function on an annulus. It is then known for any doubly connected region in terms of the conformal map to an appropriate annulus. 
Theorem 1. Given an annulus with inner and outer radii $\mu$ and 1 respectively, the propertiesunique Green's function defined on the annulus and characterized by (2)-(6) is given by

$$
G(z, w)=\Phi(z, w)+\sum_{m=-\infty}^{\infty} c_{m}(\rho, w) e^{i 2 \pi m \sigma}
$$

with $z=\rho e^{i 2 \pi \sigma}, i=\sqrt{-1}, \mu \leq \rho \leq 1$, and $0 \leq \sigma \leq 1$, where

$$
\Phi(z, w)=\log \left|(z-w) /\left(1-\frac{\mu^{2}}{z \bar{w}}\right)\right|
$$

$c_{0}(\rho, w)=a_{0}(w)$ and

$$
c_{m}(\rho, w)=\frac{(\mu / \rho)^{m}-(\mu / \rho)^{-m}}{\mu^{m}-\mu^{-m}} a_{m}(w), \quad m \neq 0
$$

with Fourier coefficients $a_{m}(w)$ defined by

$$
a_{m}(w)=-\int_{0}^{1} \Phi\left(e^{i 2 \pi \tau}, w\right) e^{-i 2 \pi m \tau} d \tau
$$

Consider now the complex potential for potential flow past an interior obstacle in a channel. We find such a complex potential using conformal mapping. The physical channel is mapped, through an annulus, to another channel of the same height but with an interior slit parallel to the walls (see Fig. 2). If these conformal maps are known, it suffices to find the appropriate complex potential on the annulus.

Assuming $g_{0}(w)$ is the conformal map from the annulus to the second channel described above, the complex potential for the channel, without considering circulation, is given by $V_{\infty} g_{0}(w)$ where $V_{\infty}$ is the uniform velocity at infinity. Therefore the streamfunction for the flow with circulation can be written as $\operatorname{Im}\left(V_{\infty} g_{0}(w)\right)+\gamma_{0} \eta_{0}(w)$ for some constant $\gamma_{0}$, where $\eta_{0}$ is harmonic, $\eta_{0}(w)=1$ on $|w|=\mu, \eta_{0}(w)=0$ on $|w|=1$. Since $\eta_{0}(w)=(\log \mu)^{-1} \operatorname{Im}(i \log w)$, setting $\gamma=-\gamma_{0} / \log \mu$ we get the desired complex potential

$$
P(w)=V_{\infty} g_{0}(w)-i \gamma \log w
$$

$P$ is unique up to an additive complex constant if the circulation is given. $g_{0}(w)$ can be calculated, in principle, using (11). However, a simple expression is also available (see e.g. [1]):

$$
g_{0}(w)=(H / \pi)\left\{\log \left[\theta\left(w / \mu w_{01}\right)\right]-\log \left[\theta\left(w / \mu w_{02}\right)\right]\right\}
$$

where $H$ is the height of the channels and $w_{01}$ and $w_{02}$ are the points on the annulus mapped to the points at infinity in the channels. Our computational domain is taken to be the annulus and all relevant formulae are defined there.

A vortex position in a flow with one vortex is in equilibrium if

$$
\frac{\partial W}{\partial w}-\frac{\kappa^{2}}{4} \frac{f^{\prime \prime}(w)}{f^{\prime}(w)}=0
$$

where $W(w)=\Psi_{0}(w)+(\kappa / 2) g(w, w), \Psi_{0}(w)=\operatorname{Im}(P(w)), P$ is given by (13)and $f$ is the conformal map from the annulus to the flow domain. Let $\Psi(\zeta, w)$ be the streamfunction (transplanted to the 
annulus) when there is a vortex at $f(w)$. If $\zeta_{0}$ maps to a sharp edge of the obstacle, then, because of the singularity of the transformation, a Kutta condition at $\zeta_{0}$ requires

$$
\left.\frac{\partial}{\partial \zeta} \Psi(\zeta, w)\right|_{\zeta=\zeta_{0}}=0
$$

If we write

$$
\frac{\partial}{\partial \zeta} \Psi(\zeta, w)=h_{1}(\zeta, w)-\frac{\gamma}{2 \zeta}+\kappa h_{2}(\zeta, w)
$$

then (15) becomes

$$
\gamma=2 \zeta_{0}\left(h_{1}\left(\zeta_{0}, w\right)+\kappa h_{2}\left(\zeta_{0}, w\right)\right) .
$$

The functions $h_{1}$ and $h_{2}$ can be determined from (7), (12) and (13). To find an equilibrium position with the Kutta condition imposed at $\zeta_{0}$, one can prescribe $\kappa$ and solve (14) (two real equations) for $w$ numerically, with $\gamma$ determined by (16). Similarly, one can impose a Kutta condition at two different points $\zeta_{1}$ and $\zeta_{2}$, determining both $\gamma$ and $\kappa$ from the system of two linear equations obtained by replacing $\zeta_{0}$ in $(16)$ by $\zeta_{1}$ and $\zeta_{2}$.

A stationary point $z^{*}$ of the system 1 is stable if the determinant

$$
\left|\begin{array}{ll}
\tilde{W}_{x x} & \tilde{W}_{x y} \\
\tilde{W}_{x y} & \tilde{W}_{y y}
\end{array}\right|=4\left(\tilde{W}_{z \bar{z}}-\left|\tilde{W}_{z z}\right|^{2}\right)>0
$$

where $\tilde{W}$ is the Routh function defined on the physical domain. (17) is equivalent to requiring that

$$
\hat{W}_{w \bar{w}}-\left|\hat{W}_{w w}\right|^{2}>0
$$

at $w^{*}=f^{-1}\left(z^{*}\right)$, where

$$
\hat{W}(w)=W(w)-\left(\kappa^{2} / 2\right) \log \left|f^{\prime}(w)\right| .
$$

Numerical differentiation was used to compute the left-hand-side of (18).

In the case of two vortices, the system of four real equations determining critical points of $W$ is again solved numerically in the parameter domain with $\kappa_{1}$ and $\kappa_{2}$ prescribed. Again $\gamma$ can be eliminated by expressing it in terms of $w_{1}$ and $w_{2}$ using the Kutta condition at the trailing edge. For the stability analysis, we calculate the eigenvalues of the Hermitian matrix $D^{2} \hat{W}\left(w_{1}, w_{2}\right)$. The vortex location is stable if all eigenvalues are positive. The entries of $D^{2} \hat{W}\left(w_{1}, w_{2}\right)$ were computed using numerical differentiation and a numerical routine was used to compute the eigenvalues.

In all the computations described below, the conformal mapping was determined using the numerical package DSCPACK [3]. In solving the system (14) numerically, the truncated series in the expressions must be evaluated efficiently. We compute Fourier coefficients using an FFT routine and evaluate the truncated series with Horner's rule. It can be easily seen that for sufficiently large $m$, terms in these series have order $O\left(\rho^{m}\right), \rho=|w|$. So in general, $2^{5}$ or $2^{6}$ Fourier points should be enough to guarantee 6 to 8 digit accuracy if $\rho$ is not too close to 1 .

All computations were done in double precision arithmetic on an IBM E59121 Model 440 at Wichita State University. In general, a fairly rough initial guess for the solution to the nonlinear system converged in a few seconds for all geometries we have tried. In order to get equilibrium positions near corners or tips of an obstacle for small strengths, points in the annulus close to prevertices usually provide good initial guesses. 


\section{Numerical results}

In the following examples we use $\preceq$ to denote "approximately less than or equal to". $z^{*}$ or $z_{i}^{*}$ will denote the location of the vortex. $V_{\infty}$ is taken to be unity. In each example the flow domain $R$ is a channel with an interior obstacle. The channel that was used in our examples, except for the last one, has parallel walls and is symmetric with respect to the origin with height $H$. In determining the doubly connected Schwarz-Christoffel map, five vertices are prescribed for the channel, two on the upper wall, one on the lower wall, plus two vertices at infinity, with additional vertices to determine the obstacle.

\subsection{Inclined flat plate}

The flat plate was taken to be of unit length and to be placed symmetrically about the origin with certain angle of attack $\alpha$. The Kutta condition was imposed at the trailing edge.

First, we compared our results with results for the same obstacle in the entire space. In the full space, equilibrium locations can be determined analytically by the method described by Saffman and Sheffield in [6]. For $H=19.6, \alpha=6^{\circ}$, and $\kappa=3 / \pi$, the equilibrium points above the leading and trailing edges, respectively, are (rounded to the fourth decimal place)

$$
z_{\text {leading }}=(-0.2234,0.3832), \quad z_{\text {trailing }}=(0.2080,0.2477),
$$

In the full space, the corresponding equilibrium positions are $(-0.2233,0.3838)$ and $(0.2076,0.2484)$. For channel flow an additional equilibrium position is found near the bottom of the channel at $(-3.0953,-8.9989)$, which has no counterpart if the walls are absent.

With $H$ as specified above, there are stable equilibrium positions when $0<\alpha \preceq 7.8^{\circ}$ for the channel, while for the entire space, there are stable equilibria for $0<\alpha \preceq 7.9^{\circ}$. In both cases, no positions in equilibrium have been found when the angle of attack is negative and $\kappa>0$. Fig. 3 gives the configuration with the equilibrium vortex position above the trailing edge (comparing with Fig. 5 in $[6])$.

Typically for $\alpha$ in the range specified above, three loci of equilibrium positions were found. A typical case is shown in Fig. 4. For the two loci above the obstacle there are equilibrium positions for all $\kappa>0$, with $\kappa \simeq 0$ near the obstacle. Positions on the locus starting from the trailing edge are stable for $\kappa$ greater than a certain minimum value depending on $H$ and $\alpha$, while the positions on the locus starting from the leading edge are always unstable. For the locus below the obstacle there is an upper bound for $\kappa$ beyond which no equilibrium positions can be found and a certain range of this locus contains stable positions. In particular, for $H=3$ and $\alpha=5^{\circ}$, this locus ends with $\kappa \simeq 7.5$ and the vortex locations for the range $0.35 \preceq \kappa \preceq 0.75$ are stable. We found that, for fixed $\alpha$ and $\kappa, H$ must be bigger than a certain value in order for an equilibrium position below the obstacle to exist. Two typical configurations corresponding to $H=2$ and $\alpha=6^{\circ}$ are shown in Fig. 5 and Fig. 6.

We also investigated the influence of angle of attack on the stability of equilibrium positions. The general conclusion is that for the equilibrium positions above the trailing edge, increasing angle of attack, with fixed $H$ and $\kappa$, will worsen the stability in the sense that an equilibrium position could lose its stability with an increased angle of attack. We also observed that for the equilibrium positions below the obstacle, the angle of attack has the opposite effect, as far as stability is concerned, i.e. increasing $\alpha$ will strengthen the stability.

The numerical results showed also that for fixed $\kappa$, the narrower the channel is, the smaller the interval of $\alpha$ will be in which the trapped equilibrium positions above the leading edge are stable. For example, with $\kappa=3 / \pi$, the height of the channel must be at least 0.95 in order for the trapped 
equilibrium positions to be stable for some positive angle of attack, while to guarantee stability of the trapped equilibrium positions for $\alpha=5^{\circ}$ and $\kappa=3 / \pi$, the height of the channel must be no less than 2.225 .

If we impose the Kutta condition at both leading and trailing edges both $\kappa$ and $\gamma$ are determined. The computed $\kappa$ is negative (positive) when an equilibrium position is above (below) the obstacle. The equilibrium position is, in general, unstable except when it is above the trailing edge and $H$ is small enough ( $\alpha$ fixed). For example, for $\alpha=5^{\circ}, H$ must be approximately less than 0.875 to have a stable solution.

\subsection{An inclined flat plate with a spoiler}

The flat plate is the same as above except that a spoiler of length 0.1 perpendicular to the plate is placed above the plate $20 \%$ forward of the trailing edge. The Kutta condition was again imposed at the trailing edge. Fig. 7 gives an example with $\mathrm{H}=2, \alpha=5^{\circ}$, and $\kappa=3 / \pi$. The corresponding configuration remains stable for $0<\alpha \preceq 21$. $^{\circ}$ for equilibrium points on the same locus.

We have found that by varying $\kappa$ with fixed $H$ and $\alpha$, the number of equilibrium loci and their locations will depend on the location, length, and inclination of the spoiler. In particular, we kept the spoiler to be of length 0.1 and perpendicular to the plate and investigated two different locations. For the spoiler described at the beginning of this example $H=3$ and $\alpha=5^{\circ}$, we found four loci above and one below the obstacle (see Fig. 8). Equilibrium points on the portion $0 \prec \kappa \preceq 12.782$ of the locus starting from the left re-entrant corner are stable. The equilibrium positions below the obstacle corresponding to $0.4 \preceq \kappa \preceq 0.95$ are also stable. (As in example (1), for fixed $\kappa$, stationary positions can be found only for $H$ greater than a certain value.) The remaining equilibrium positions are unstable.

With the same $H$ and $\alpha$, we then place the same spoiler at the same distance from the leading edge as it was previously from the trailing edge. Five loci above the obstacle were found as shown in Fig. 9. (There are also loci below the obstacle not shown on the graph). There are three stable loci, two starting from the re-entrant corners and one starting from the trailing edge.

We have also investigated how the length of the spoiler affects the location of loci. When the length was reduced to approximately 0.06 , the locus above the tip of the spoiler disappeared (see Fig. 8) and a locus starting from the trailing edge was found instead. This was expected since for very small spoiler, the results should be very close to those for the plate without spoiler. While the locus above the tip of the spoiler didn't change much, increasing the length of the spoiler caused the two loci on the left of the spoiler to become closer. We also observed that reducing the height of the channel forced these two loci to be further separated.

Finally, we mention briefly that we also tried to impose the Kutta condition at both the tip of the spoiler and the trailing edge. For the equilibrium points above (below) the obstacle, $\kappa$ was found to be positive (negative). The equilibrium vortex above the leading edge is much stronger than the one above the trailing edge $(\kappa=1.3821$ and $\kappa=0.0765$ respectively $)$.No stable equilibrium positions were found.

\subsection{NASA 23024 airfoil}

The airfoil is placed in a channel with height 2 and $\alpha=5^{\circ}$. The configuration is given in Fig. 10. Fig. 11 shows all loci of equilibrium positions that we found with positive $\kappa$ 's. For the channel flow, again an equilibrium locus near the bottom of the channel was found. The ranges of $\kappa$ used to plot loci are the followings: $\kappa \in[0.01,20$.$] for the locus near the leading edge; \kappa \in[0.07,20$.$] for the$ locus near the trailing edge; $\kappa \in[0.77,30$.$] for the locus below the trailing edge; and \kappa \in[0.01,20$. 
for the one near the bottom of the channel. Portions of the locus near the trailing edge and the locus near the bottom of the channel are stable.

\subsection{Flow with two point vortices}

A flat plate with an attached spoiler of length $0.15,30 \%$ forward of the trailing edge was chosen. The Kutta condition was imposed at the trailing edge and $\alpha=5^{\circ}$. For this obstacle, five typical equilibrium configurations are shown in Figs. 12-14. Those in Figs. 12 and 13 are stable.

It is shown in Miller [5] that if the boundary of a flow domain has convex corners, then there is a configuration of stable point vortices with a vortex near each convex corner, and, moreover, there are configurations with a finite sequence of vortices of alternating sign in each corner. Figure 13 is an example of a stable configuration with a vortex on each side of the spoiler. Figure 15 shows a stable configuration with two vortices of opposite sign on one side of the spoiler. (The vicinity of the vortices is enlarged.)

For the flat plate without spoiler, no equilibrium pairs could be found on the same side of the plate. (We did, however, find pairs separated by the plate.) We also tried other geometries. Our numerical results support the conjecture that if two vortices are in stable equilibrium with respect to a convex obstacle in a channel with parallel walls, then the line segment connecting the vortices must intersect the obstacle.

\subsection{A channel with a bump on the bottom}

A plate inclined at of angle of attack $\alpha=5^{\circ}$ is placed in a channel $(H=2)$ with a circular bump on the bottom. The semi-circle (with center -0.5 and radius 0.5 ) is approximated by a polygon. $\kappa=1$ and the Kutta condition was imposed at the trailing edge. There are four stable equilibrium vortex positions (two above and two below the trailing edge). They are $(0.3940,0.2107)$ (the corresponding configuration is shown in Figure 16), (0.3462, 0.1403), (0.3940,-0.5235), and $(0.2709,0.6620)$. There are at least four additional unstable equilibrium positions, two above and two below the leading edge.

We note that any stable configuration of point vortices can be desingularized ([2]).

\section{References}

[1] Daeppen, H.D., Die Schwarz-Christoffel-abbildung fur zweifach zusammenhangende gebiete mit anwendungen, Ph.D. diss., ETH, Zurich, 1988.

[2] Elcrat, A.R. and Miller, K.G., "Rearrangements in Steady Multiple Vortex Flows," Comm. in PDE, 20, pp. 1481-1490, 1995.

[3] Hu, C., User's Guide to DSCPACK, NIAR Report 95-1, National Inst. for Aviation Research, Wichita, KS, 1995.

[4] Lin, C.C., "On the Motion of Vortices in Two Dimensions," Univ. Toronto Appl. Math. Series, No. 5, Toronto Univ. Press, 1943.

[5] Miller, K.G., "Stationary Corner Vortex Configurations," Z. angew. Math. Phys., 47, pp. 39-56, 1996.

[6] Saffman, P.G. and Sheffield, J., "Flow Over a Wing With an Attached Free Vortex," Studies in Applied Math., 57, pp. 107-117, 1977. 

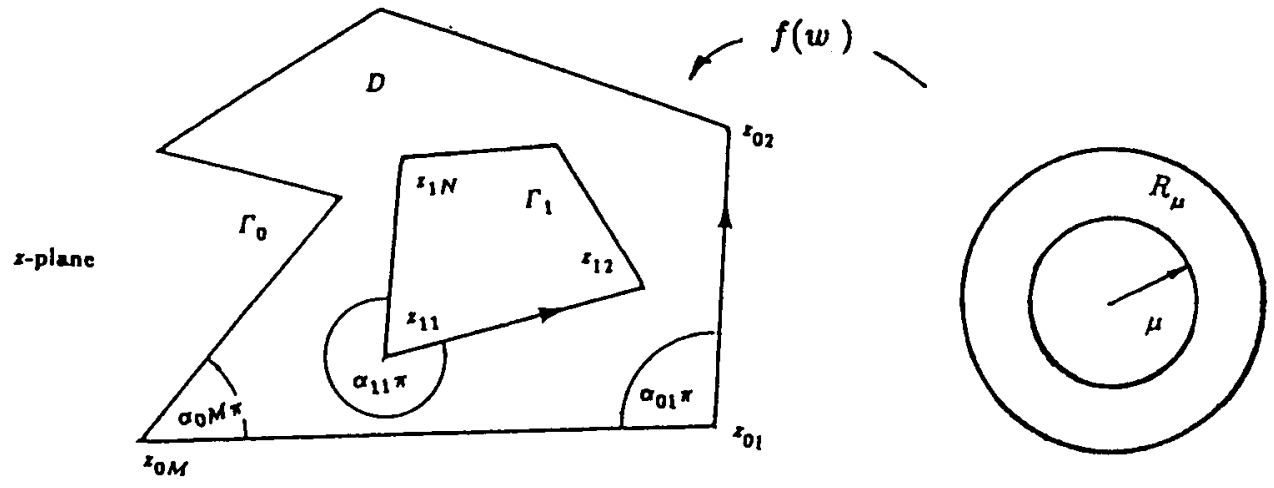

Figure 1: Notation relevant to the doubly connected Schwarz-Christoffel transformation.

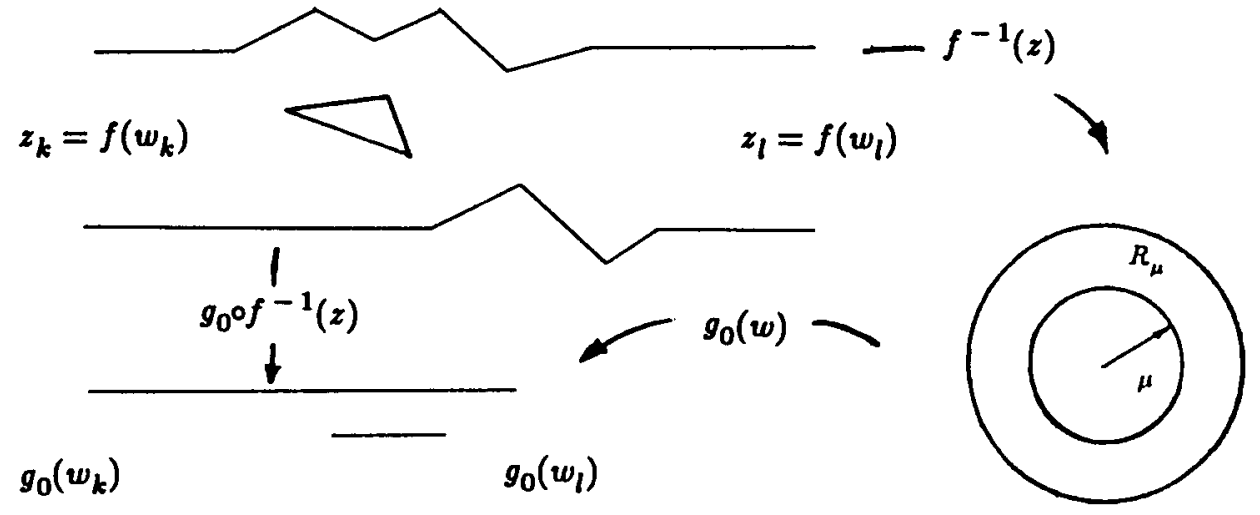

Figure 2: Mapping procedure from the physical channel to the model channel.

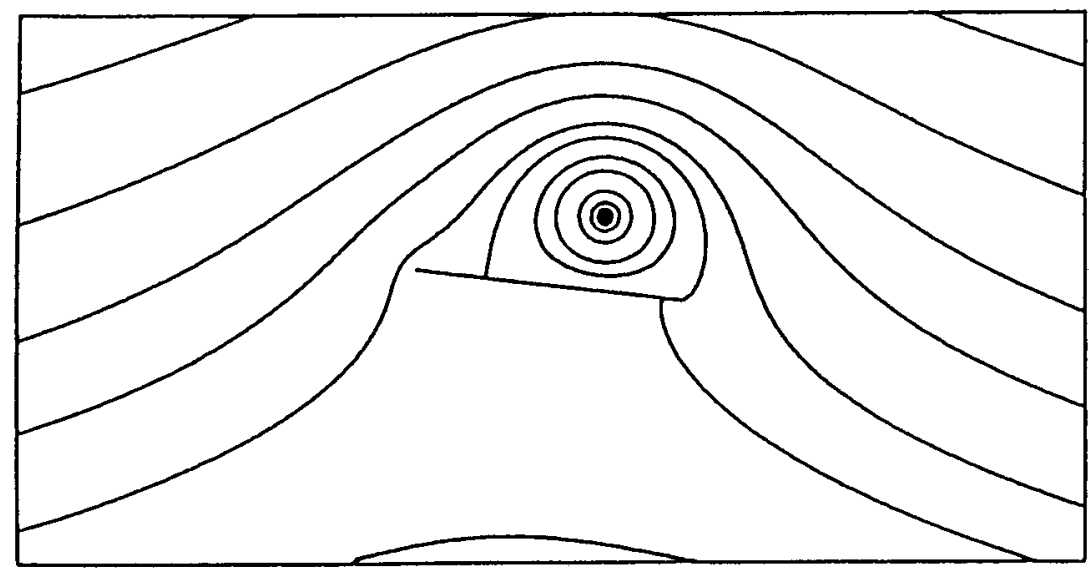

Figure 3: Flow past a plate: $\mathrm{H}=19.6, \alpha=6^{\circ}$, and $\kappa=3 / \pi$. 


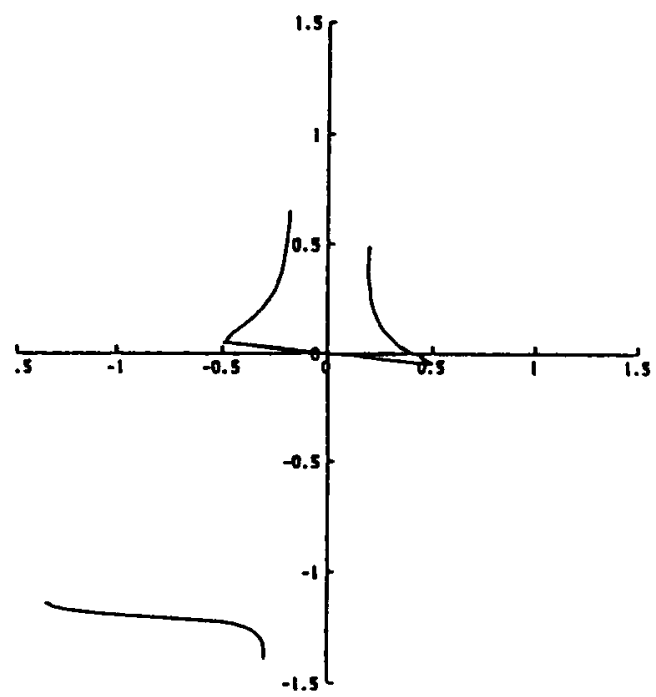

Figure 4: Flow past a plate: three loci of equilibrium points for $\mathrm{H}=3, \alpha=5^{\circ}$.

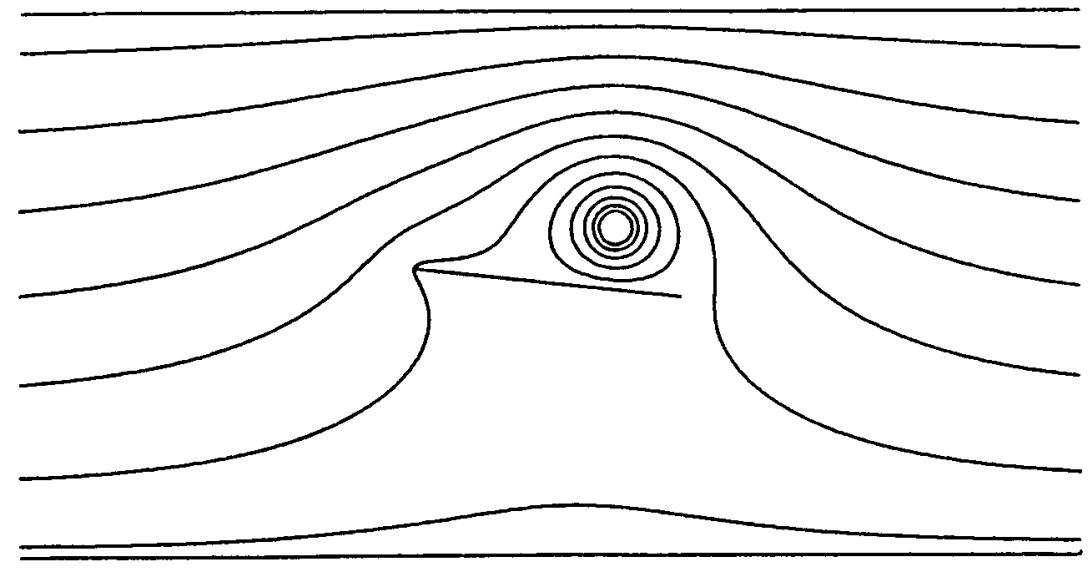

Figure 5: Flow past a plate with vortex attached to the plate: $\mathrm{H}=2, \alpha=6^{\circ}$ and $\kappa=1$.

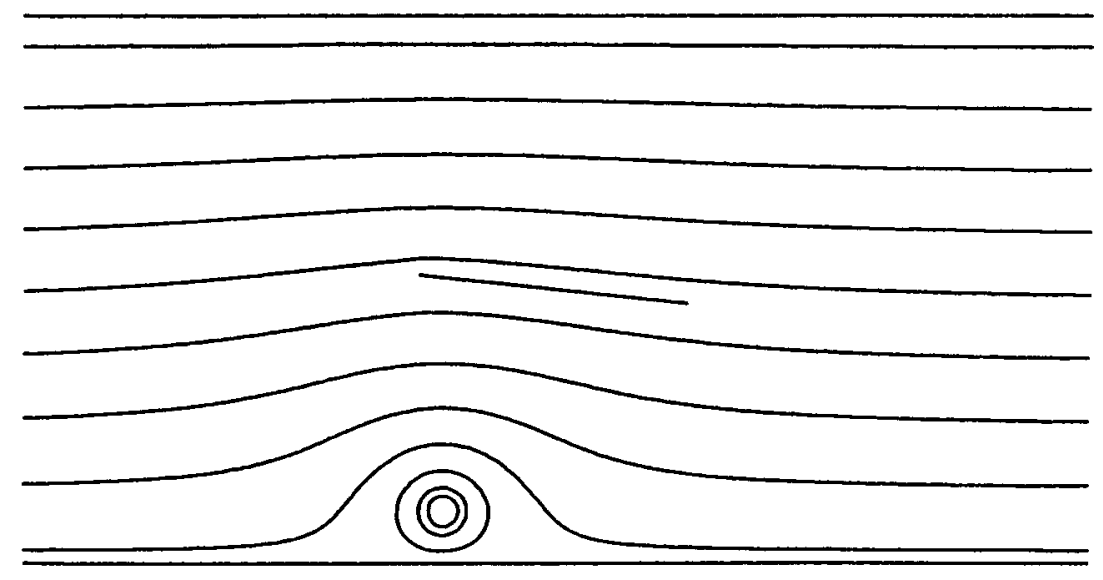

Figure 6: Flow past a plate with vortex attached to the wall: $\mathrm{H}=2, \alpha=6^{\circ}, \kappa=.4$. 


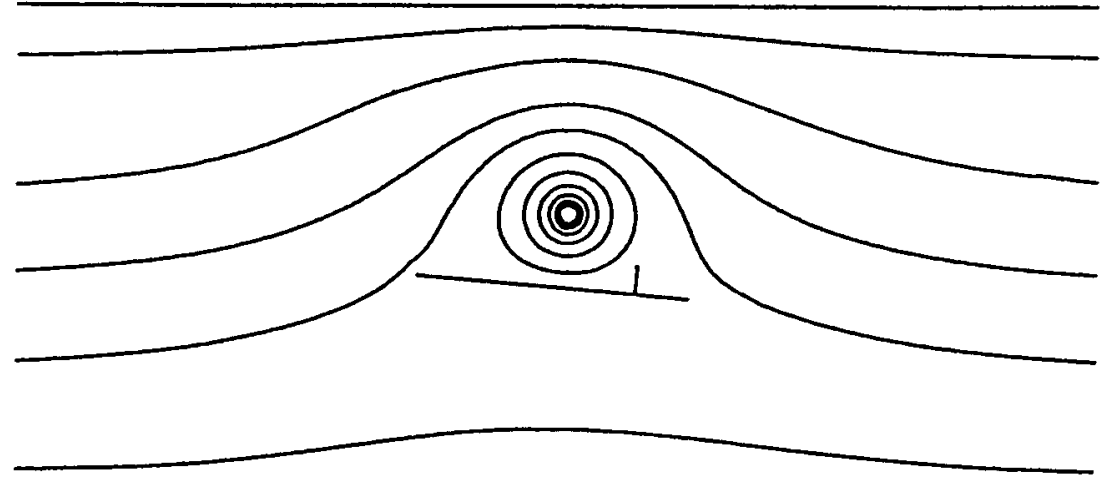

Figure 7: Flow past a plate with a spoiler: $\mathrm{H}=2, \alpha=6^{\circ}, \kappa=3 / \pi$.

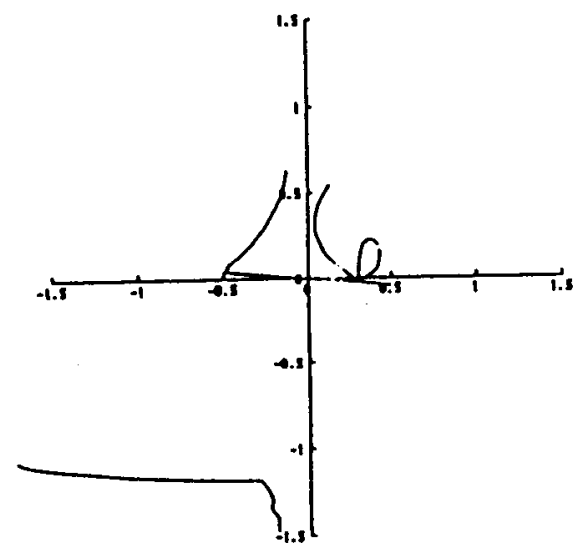

Figure 8: Four loci of equilibrium points for flow past a plate with spoiler located $20 \%$ forward of trailing edge. $\mathrm{H}=3, \alpha=5^{\circ}$. 


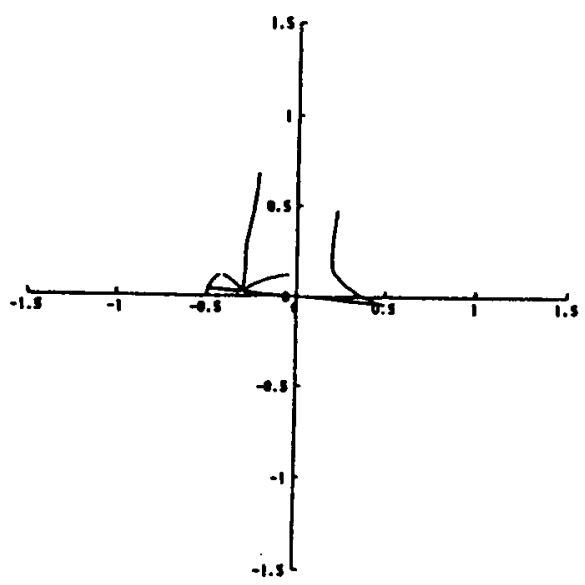

Figure 9: Five loci of equilibrium points for flow past a plate with spoiler located $20 \%$ back of leading edge. $\mathrm{H}=3, \alpha=5^{\circ}$.

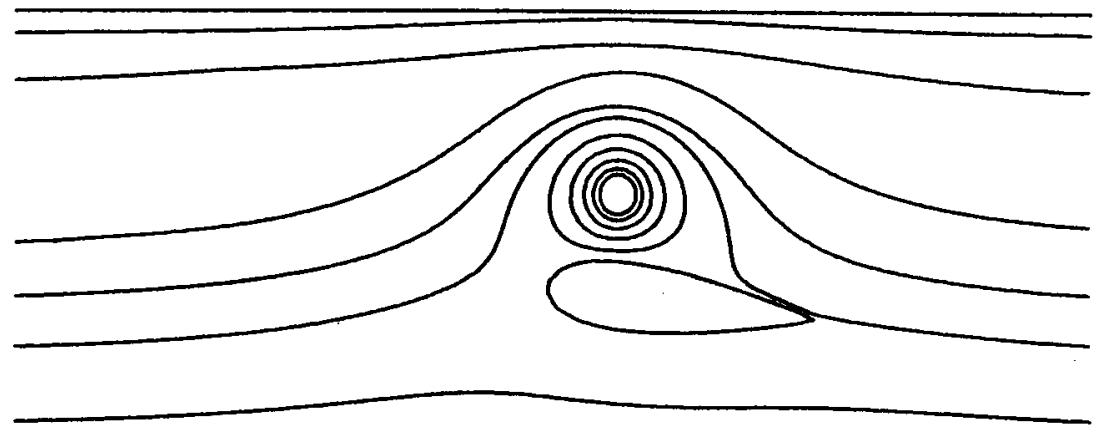

Figure 10: Flow past an airfoil: $\mathrm{H}=2, \alpha=5^{\circ}, \kappa=.9$. 


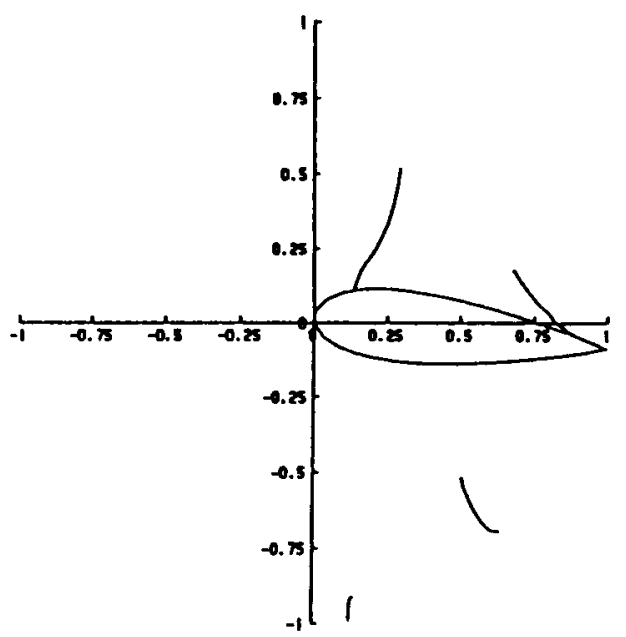

Figure 11: Four loci of equilibrium points for flow past an airfoil: $\mathrm{H}=2, \alpha=5^{\circ}$.

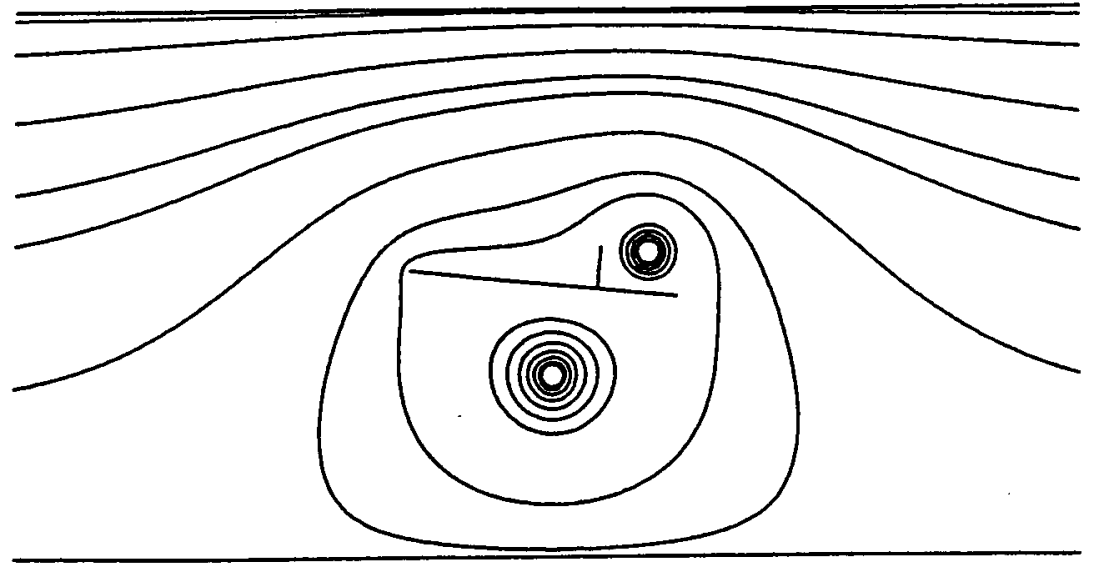

Figure 12: Flow past a plate with a spoiler and two vortices: $\mathrm{H}=2, \alpha=5^{\circ}, \kappa_{1}=\kappa_{2}=1$.

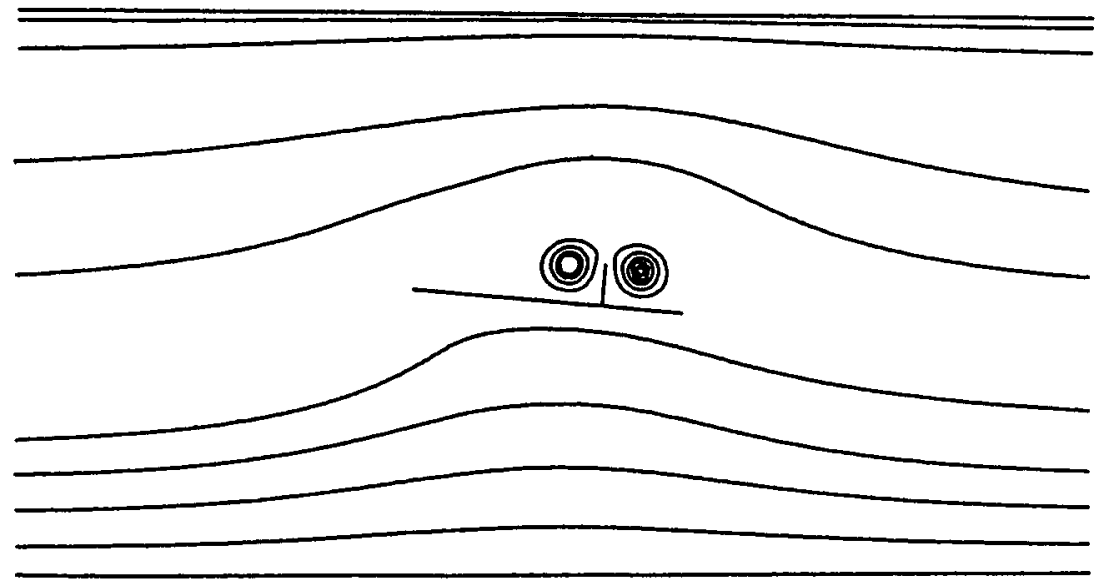

Figure 13: Flow past a plate with a spoiler and two vortices: $\mathrm{H}=2, \alpha=5^{\circ}, \kappa_{1}=\kappa_{2}=.35$. 


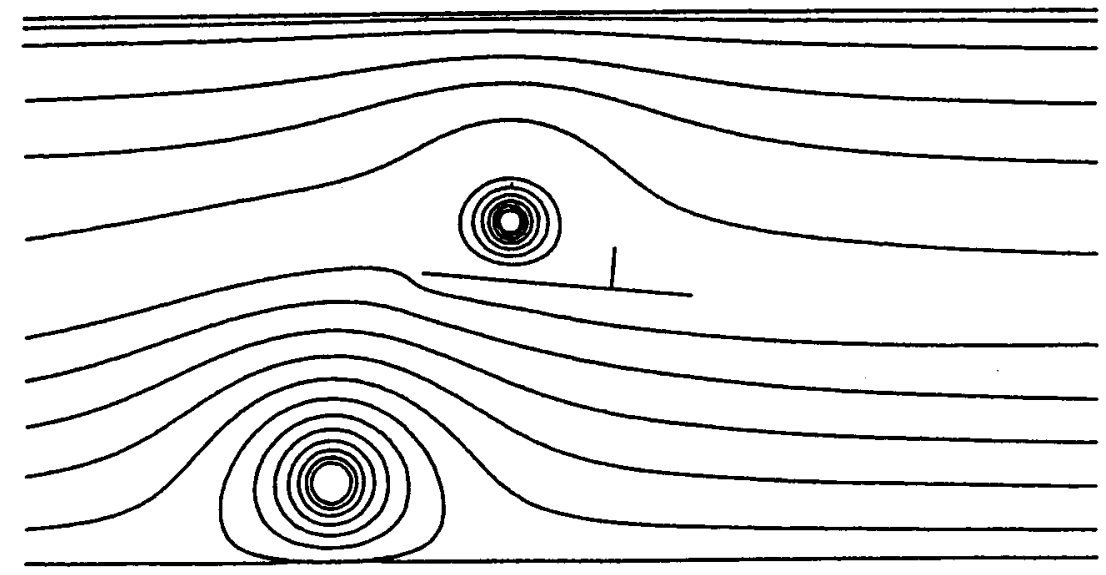

Figure 14: Flow past a plate with a spoiler and two vortices: $\mathrm{H}=2, \alpha=5^{\circ}, \kappa_{1}=\kappa_{2}=0.6$.

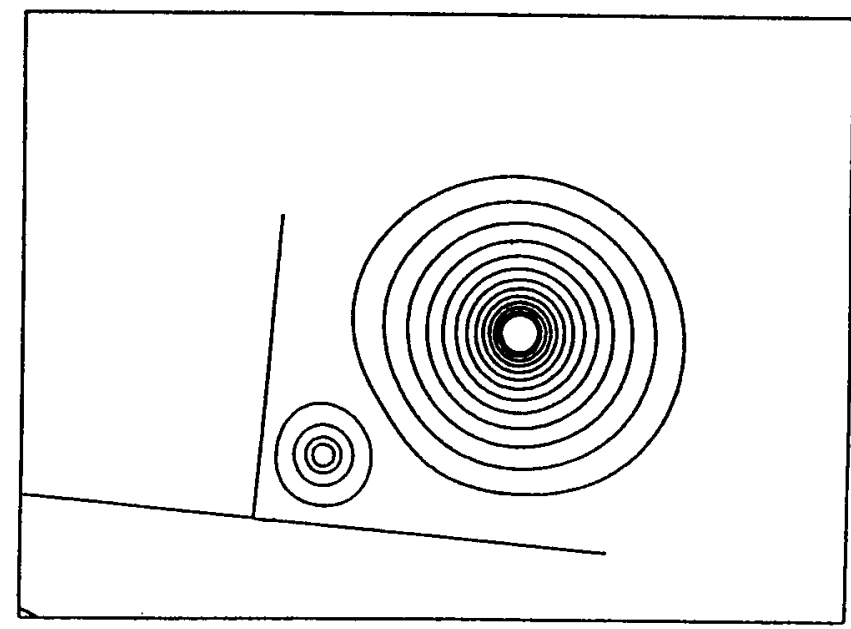

Figure 15: Flow past a plate with a spoiler and two vortices: $\mathrm{H}=2, \alpha=5^{\circ}, \kappa_{1}=-0.5, \kappa_{2}=1.5$.

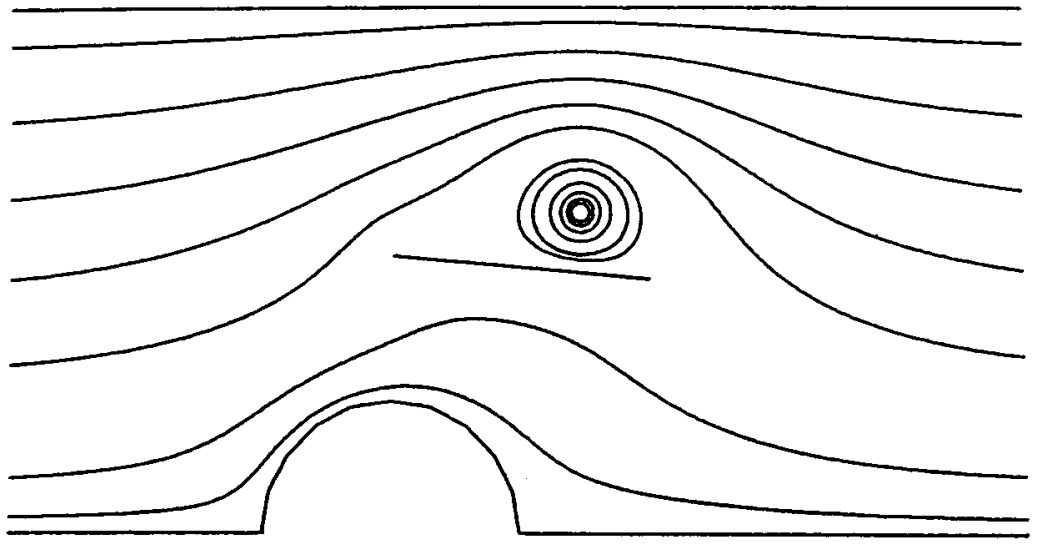

Figure 16: Flow past a flat plate with a circular obstruction on the wall of the channel. 